Original Research Paper

\title{
Building Probability Functions by Persian Curve
}

\author{
${ }^{1}$ Abdolrasoul Ranjbaran, ${ }^{2}$ Mohammad Ranjbaran and ${ }^{3}$ Fatema Ranjbaran \\ ${ }^{I}$ Department of Civil and Environmental Engineering, Shiraz University, Iran \\ ${ }^{2}$ Parsian Azad University, Iran \\ ${ }^{3}$ Department of Mechanical Engineering, Shiraz University, Iran
}

\author{
Article history \\ Received: 30-08-2020 \\ Revised: 13-10-2020 \\ Accepted: 29-10-2020 \\ Corresponding Author: \\ Abdolrasoul Ranjbaran \\ Department of Civil and \\ Environmental Engineering, \\ Shiraz University, Iran \\ Email: ranjbarn@shirazu.ac.ir \\ aranjbaran@yahoo.com
}

\begin{abstract}
Construction of a probability function for a given random data is the main theme of several branches of human knowledge, where it is an active area of study. In spite of great developments, the solution needs extensive effort and the results contain epistemic uncertainty. In this study, making use of the logical reasoning and concise mathematical logics, a method called the change of state philosophy, which is digested in the Persian curves, is derived. The Persian curves that have the necessary and sufficient condition for a probability function, are explicitly derived, via the ordinates of four specific points on the random data. The proposed Persian curves are free of epistemic uncertainty and their flexibility provide the possibility for the insertion of the expert's will. The work is validated via concise logical formulation and comparison of the results with those of the others.
\end{abstract}

Keywords: The Change of State Philosophy, Persian Curves, Probability, Phenomenon Functions, State Functions, State Variable

\section{Introduction}

The likelihood value of a change in a system, is conventionally defined as probability. A phenomenon is conceived as a change in the system. Diversity in the phenomena concluded in the diversity of probability distributions for their statistical analysis. The Probability function is present in problems containing uncertainty and have a long history in mathematics and engineering which dates back to the 17th century (David, 1962). From that time on a well-established probability theory have been developed, in all areas of human knowledge. A statistical distribution function of wide variability is proposed by (Weibull, 1951), which became very popular probability mathematical distribution for its relative flexibility in modelling lifetime data. But it does not provide a reasonable parametric fit for modelling phenomena with non-monotone data. Because of its importance, the probability analysis is applied in different fields of study (David, 1962; Williamson, 1989; Gouda, 2005; Huang, 2009; Amusan, 2010; Butler, 2011; Cui, 2011; Andrews, 2012; Adamski, 2014; Hao, 2014; Heinemann and Ohm, 2014; Adefisoye, 2015; Alghamdi, 2015; Ali, 2016; Perrone, 2018). In spite of great advance in this field, the work is not complete. This can be observed from the ongoing investigations in this days. For example, novel functional representation for the Probability Density Functions (PDF) of random variables was constructed and efficient and accurate algorithms for computing the (PDF) of their sums, products and quotients were developed by (Satkauskas, 2017). Or (Boehm, 2019) evaluated the performance of expected shortfall estimation with normal, student-t and skewed distributions. The conclusion is restricted to financial returns and forecasting expected shortfalls. Conventionally, for statistical analysis of a phenomenon, a distribution will be selected, with logically no approved relation between the phenomenon and the selected function. Therefore the probability functions in the literature, introduce epistemic uncertainty to the work. The era is not finished, because the conventional probability has a weak logical basis. The main aim of the presented work is to propose a certain probability function, based on sound mathematical foundation. Toward the aim, in the first two decades of 21st century, our research team, conducted an extensive research for analysis of phenomena which are partly published in references (Amirian and Ranjbaran, 2019; Baharvand and Ranjbaran, 2020a-b; Ranjbaran et al., 2011; 2013; 2020a-b; Ranjbaran and Rousta, 2013; Ranjbaran and Ranjbaran, 2014; 2016; 2017a-b; 2018; Hoseini et al., 2018; Ranjbaran, 2010; 2014). More detailed information may be obtained in (Ranjbaran et al., 2020b). Via logical reasoning and concise mathematics their research concluded in the so called change of state philosophy. The change of state philosophy is digested into the Persian curves. In view of the vocabulary of the statistics the Persian curves are the probability distribution which 
are exact entities that should be used in place of the conventional likelihood value. Detail of formulation is presented in the next section.

\section{Basic Formulation}

In this section, the basic formulation for the change of state philosophy via a sound logical basis, is developed. The traditional formulations in the academic universe are divided into the stiffness method and the flexibility method. The so called stiffness method, is based on the change of a system capacity, called the stiffness, which reduces to zero during the change of state. While the flexibility method makes use of the flexibility, i.e., inverse of stiffness, which goes toward the infinity during the change of state. Near the end of the phenomenon, the stiffness become very small and the flexibility become very large and hence introduce error into the work. On the other hand the change of state philosophy uses the equalities, $\left(k_{S S} \times f_{S F}=1\right)$ and $\left(k_{S} \times f_{S}=1\right)$, as shown in Fig. 1 , which are free of epistemic uncertainty. The equalities is then expressed as equality of $\left(k_{S S} \times f_{S}\right)$ and $\left(k_{S} \times f_{S F}\right)$ in Equation (1), in which $\left(k_{S S}=k_{S^{-}} k_{C}\right)$ is the survivedstiffness, $\left(f_{S F}=f_{S}+f_{C}\right)$ is the survived-flexibility, $\left(k_{S}\right)$ is the system-stiffness, $\left(f_{S}\right)$ is the system flexibility, $\left(k_{C}\right)$ is the change-stiffness and $\left(f_{C}\right)$ is the change-flexibility:

$k_{S S} / k_{S}=f_{S} / f_{S F} \quad\left(k_{C}\right) \&\left(f_{C}\right)=?$

Equation (1) is rearranged to obtain the $\left(k_{S S}\right)$ and $\left(k_{C}\right)$, in terms of the other parameters, in Equation (2):

$$
\begin{aligned}
& k_{S S}=\frac{f_{S} \times k_{S}}{f_{S}+f_{C}}=\frac{f_{S}}{f_{S}+f_{C}} \times k_{S}=S_{R} \times k_{S} \\
& k_{C}=k_{S}-k_{S S}=\frac{f_{C}}{f_{S}+f_{C}} \times k_{S}=F_{R} \times k_{S}
\end{aligned}
$$

in which the phenomenon functions (collection of the failure function $\left(F_{R}\right)$ and the survive function $\left(S_{R}\right)$ ) are defined in Equation (3):

$$
F_{R}=\frac{f_{C}}{f_{S}+f_{C}} \in\left[\begin{array}{ll}
0 & 1
\end{array}\right] \quad S_{R}=\frac{f_{S}}{f_{S}+f_{C}} \in\left[\begin{array}{ll}
1 & 0
\end{array}\right]
$$

The proposed form of phenomenon functions in Equation (3) are defined in terms of the $\left(f_{S}\right)$ and the $\left(f_{C}\right)$, which are unknown to this end. The investigation for explicit definition of these functions is continued as follows via construction of the so called state functions.

Development of a functional in terms of two functions is not possible. Then the phenomenon functions are customized for $\left(k_{S}=f_{S}=1\right)$ in order to define the destination function $(D)$, the origin function $(O)$ and the state Ratio $(R)$ (which are collectively called the state functions) in Equation (4):

$k_{S}=f_{S}=1 \quad F_{R} \Rightarrow D \quad S_{R} \Rightarrow O \quad f_{C} \Rightarrow R$

Consequently the $(D \& O)$ are defined in terms of the $(R)$ in Equation (5):

$D=\frac{R}{1+R} \quad O=\frac{1}{1+R} \quad R=\frac{D}{O}$

The state functions may be considered as the solution of the boundary value problems as expressed in Equation (6) and shown in Fig. 2, where (min) denotes minimum and (max) denotes maximum:

$D=\left\{\begin{array}{ll}\min =0 & @ R=0 \\ \max =1 & @ R=\infty\end{array} \quad O= \begin{cases}\max =1 & @ R=0 \\ \min =0 & @ R=\infty\end{cases}\right.$

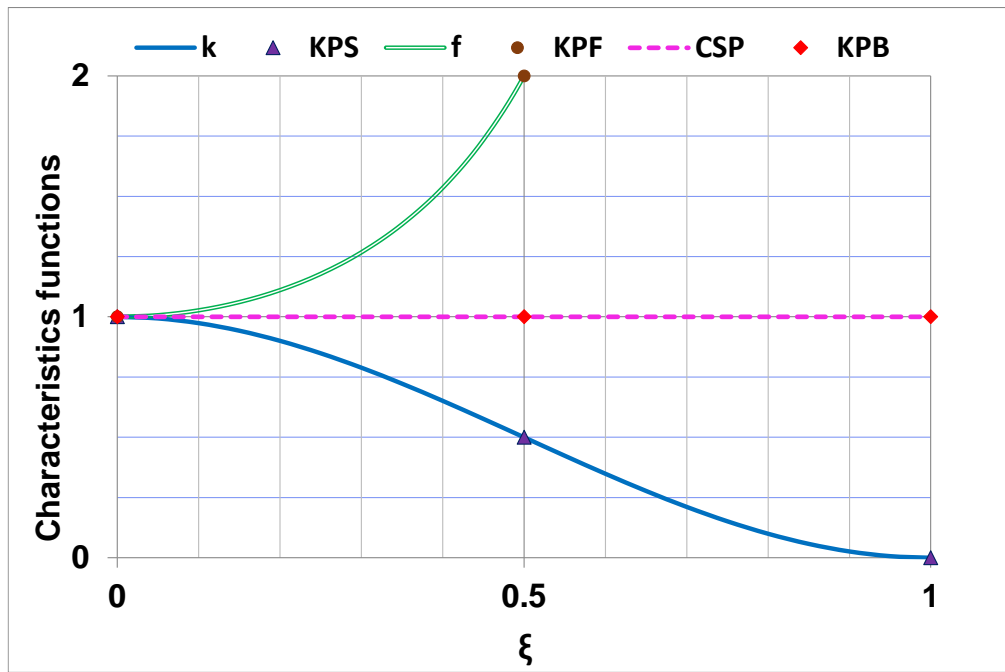

Fig. 1: Change of state philosophy basic equation 
The state ratio, with the far end in the infinity (Fig. 2), is not a good working parameter. Moreover, this ratio is itself a function, so it is not wise to be used as an independent variable. Therefore, the state variable $\xi \in[0$ 1] with a zero value $(\xi=0)$ at the origin and a unit value $(\xi=1)$ at the destination is innovatively defined. In term of the state variable, the boundary value problems in Equation (6) is rewritten as in Equation (7):

$$
D=\left\{\begin{array}{ll}
\min =0 & @ \xi=0 \\
\max =1 & @ \xi=1
\end{array} \quad O= \begin{cases}\max =1 & @ \xi=0 \\
\min =0 & @ \xi=1\end{cases}\right.
$$

Investigation for construction of solution for boundary value problems in Equation (7), led the authors to make use of their experience in structural mechanics, finite element method, mathematics and their extensive research. The results are the state functions as defined in Equation (8) and shown in Fig. 3, (Ranjbaran et al., 2020b):

$$
\begin{aligned}
& D=0.25\left(2-1+6 \xi^{2}-4 \xi^{3}-\cos \pi \xi\right) \\
& O=0.25\left(2+1-6 \xi^{2}+4 \xi^{3}+\cos \pi \xi\right)
\end{aligned}
$$

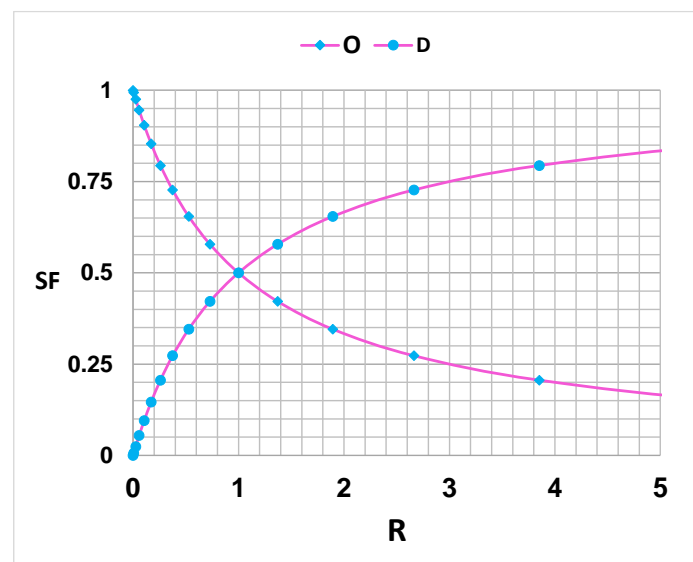

Fig. 2: State Functions versus the state Ratio (R)

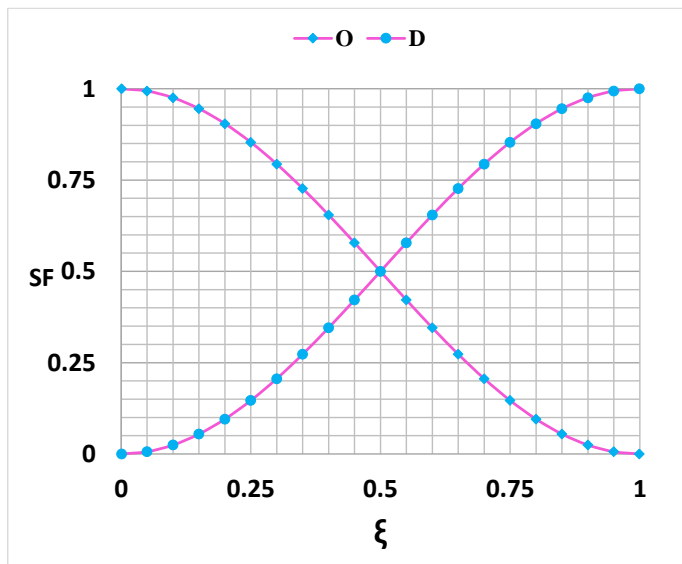

Fig. 3: State functions versus the $(\xi)$
Via the definition of the survived stiffness $\left(k_{S S}\right)$ and the survived flexibility $\left(f_{S F}\right)$, the authors detected a fact that, the $\left(f_{C}\right)$ is directly proportional to the $\left(k_{S}\right)$ ! This detection is called "the Persian Principle of Change $(P P C)$ ". In view of this principle the $\left(f_{C}\right)$ is defined in Equation (9):

$f_{C} / R=k_{S} / 1 \quad f_{C}=k_{S} R \quad f_{C}=k_{S} D / O$

Substitution of Equation (9) into Equation (3) concluded in the general definition for the phenomenon functions in Equation (10):

$F_{R}=\frac{k_{S}^{2} D}{O+k_{S}^{2} D} \quad S_{R}=\frac{O}{O+k_{S}^{2} D}$

The $\left(k_{S}\right)$ is not explicitly known and so it is not a feasible working parameter. Therefore Equation (10) is rewritten in a unified form as in Equation (11), in terms of the positive control parameters $\left(a_{M}\right)$ and $(b)$, (Ranjbaran et al., 2020b). Selection of two control parameters provided the flexibility for translation and rotation of the phenomenon functions in the $(1 \times 1)$ generic working box and let the experts to enforce their expertise to the work. The control parameters are to be determined from calibration of reliable data:

$$
F_{R}=\frac{a_{M} D^{b}}{O^{b}+a_{M} D^{b}} \quad S_{R}=\frac{O^{b}}{O^{b}+a_{M} D^{b}}
$$

To this end the proposed formulation is mathematically in an abstract form, so it is a universal one in the sense that it is independent of geometrical and material properties and the changing agent. Therefore, it applies to all natural phenomena!

As observed, the proposed formulation, were derived based on logical reasoning and concise mathematical logics. There was no need for construction and solution of differential and/or integral equations, which is the paramount basis of the conventional human knowledge. Therefore, the proposed formulation is free of epistemic uncertainty (lack of knowledge). For a given phenomenon, the lifetime is truncated at a workable interval $\left(\lambda \in\left[\lambda_{O}, \lambda_{T}\right]\right)$ and is mapped onto the state variable $(\xi \in[0,1])$ in Equation $(12)$, where $\left(\lambda_{O}\right)$ is the origin and $\left(\lambda_{T}\right)$ is the end of lifetime:

$$
\lambda=(1-\xi) \lambda_{O}+(\xi) \lambda_{T} \quad \xi=\left(\lambda-\lambda_{O}\right) /\left(\lambda_{T}-\lambda_{O}\right)
$$

In terms of the lifetime, here, the $\left(F_{R}\right)$ is renamed as Persian-Failure-curve $\left(P_{F}\right)$ and the $\left(S_{R}\right)$ is renamed as Persian-Survive-curve $\left(P_{S}\right)$ and the two collectively called the Persian-curves $\left(P_{C}\right)$. In comply with vocabulary of human knowledge, the $\left(P_{S}\right)$ is the unified 
equation for the capacity and reliability and the $\left(P_{F}\right)$ is the unified equation for the probability and fragility. The feasible (capacity and reliability) and (probability and fragility) data are managed in decreasing and increasing order respectively. For a reliable data, $\left(P_{S}\right)$ for increasing data and $\left(P_{F}\right)$ for decreasing data, is defined as the $\left(P_{C}\right)$ in Equation (13), in which $\left(P_{O}\right)$ is the ordinate of the start point $(O)$ and $\left(P_{T}\right)$ is the ordinate of the end point $(T)$ :

$$
P_{C}=\left(P_{O} O^{b}+P_{T} a_{M} D^{b}\right) /\left(O^{b}+a_{M} D^{b}\right)
$$

Moreover, in comply with the common practice in stochastic analysis, the Probability Density Function $(P D F)$ (here called the Persian-Distribution-curve $\left(P_{Z}\right)$ ), is defined as the derivative of the phenomenon functions with respect to the state variable $(\xi)$, in Equation (14), in which $\left(F_{R}^{(1)}, S_{R}^{(1)}\right.$ and $\left.D^{(1)}\right)$ are the derivatives of $\left(F_{R}, S_{R}\right.$ and $D$ ) with respect to the $(\xi)$ respectively:

$$
F_{R}^{(1)}=+P_{Z} \quad S_{R}^{(1)}=-P_{Z} \quad P_{Z}=\frac{b a_{M} D^{b-1} O^{b-1} D^{(1)}}{\left(O^{b}+a_{M} D^{b}\right)^{2}}
$$

For a reliable decreasing data as shown in Fig. 4, the sign parameter is set equal to minus $1\left(A_{ \pm}=-1\right)$ and for a reliable increasing data as shown in Fig. 5, the sign parameter is set equal to plus $1\left(A_{ \pm}=+1\right)$. Then the positive control parameters $\left(a_{M}\right)$ and (b) are obtained, in terms of the coordinates of the Key-Points (KPS), in Equation (15):

$$
\begin{aligned}
& \frac{a_{N}}{A_{ \pm}}=\frac{P_{N}-P_{O}}{P_{T}-P_{N}} \quad \frac{a_{M}}{A_{ \pm}}=\frac{P_{M}-P_{O}}{P_{T}-P_{M}} \\
& b=\frac{\log \left(a_{N} / a_{M}\right)}{\log \left(D\left(\xi_{N}\right) / O\left(\xi_{N}\right)\right)}
\end{aligned}
$$

The key points are defined as the Origin point $(\mathrm{O})$, the Middle point $(\mathrm{M})$, the Truncation point $(\mathrm{T})$ and the Next point (N) (a point between the other three), defined in Equation (16) and shown in Fig. 4 and 5, for decreasing data and increasing data respectively. Note that, points $(\mathrm{O})$ and $(\mathrm{T})$ are used for mapping in Equation (12) and Points $(\mathrm{N})$ and $(\mathrm{M})$ are used for determination of $\left(a_{M}\right)$ and $(\mathrm{b})$ in Equation (15):

$$
O\left(0.00, P_{O}\right) \quad N\left(\xi_{N}, P_{N}\right) \quad M\left(0.50, P_{M}\right) \quad T\left(1.00, P_{T}\right)
$$

Finally via logical reasoning, a unified equation for probability of failure (fragility) curve is proposed as follows. Extensive investigation of the authors research team concluded into the fact that the fragility curve for complete failure is defined in Equation (17), where $\left(P_{S U}\right)$ is unified capacity, $\left(P_{F U}\right)$ is unified fragility and $\left(P_{Z U}\right)$ is unified density. As shown, fragility is equal to $1 \mathrm{~min}$ capacity. Moreover for the horizontal axis the $(I M \in$ $[0,3])$ is replaced by the relative slenderness $(\lambda \in[0,3])$ :

$$
\begin{aligned}
& P_{F U}=\frac{1.8 D}{1+D} \quad P_{Z U}=\frac{2 D^{(1)}}{(1+D)^{2}} \quad P_{S U}=\frac{1-0.8 D}{1+D} \\
& a_{M}=2 \quad b=1
\end{aligned}
$$

Where the key point ordinate on unified Persian curves are defined in Equation (18):

$\begin{array}{ccccc}P C & O & N & M & T \\ \lambda & 0.000 & 0.750 & 1.500 & 3.000 \\ \xi & 0.000 & 0.250 & 0.500 & 1.000 \\ P_{F U} & 0.000 & 0.237 & 0.600 & 0.900 \\ P_{S U} & 1.000 & 0.763 & 0.400 & 0.100 \\ P_{Z U} & 0.000 & 1.686 & 1.364 & 0.000\end{array}$

Persian curve is managed as a bridge between the abstract mathematical concepts and the real world. Therefore it is applied for analysis of data in several branches of human knowledge in the following section. Here is the end of the proposed logical formulation. Note that the question $\left(\left(k_{C}\right) \&\left(f_{C}\right)=\right.$ ?) in Equation (1) is logically answered.

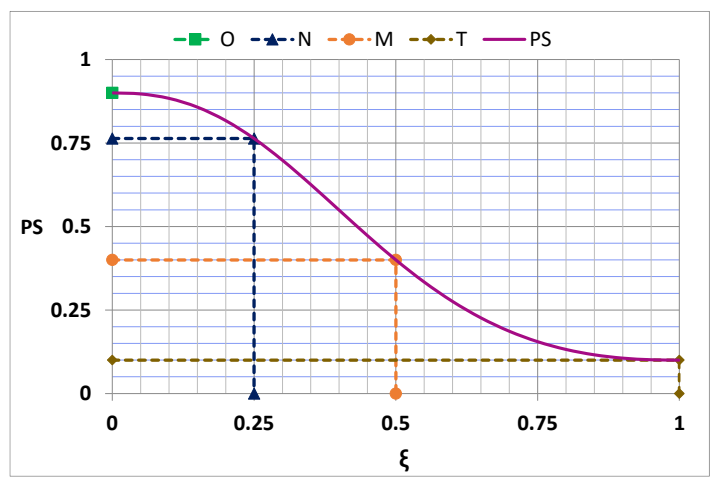

Fig. 4: Key points on persian-survive-curve

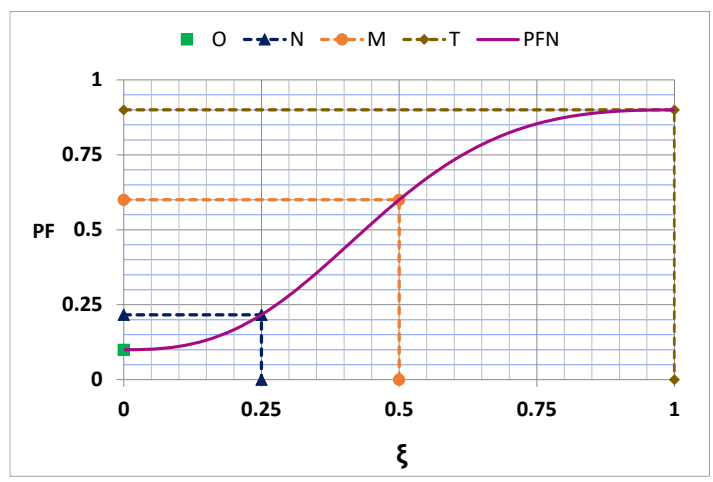

Fig. 5: Key points on persian-failure-curve 


\section{Building Probability Functions}

In view of vocabulary of probability theory in the literature, the $\left(P_{F}\right)$ is in comply with the Cumulative Distribution Function $(C D F)$, the $\left(P_{S}\right)$ is in comply with the probability of survive (reliability) and the $\left(P_{Z}\right)$ is in comply with the Probability Density Function $(P D F)$. In comply with the common practice in the literature the results are compared with those of the others in this section.

\section{Example 1}

In this example a set of data, that was analyzed in (Powley, 2013) PhD dissertation and also in (Keelin and Powley, 2011; Keelin, 2016), is selected for analysis with the Persian-curve.

Once the decision analyst asserts that he has sufficient quantile-probability data, he strives to find a continuous probability distribution that is consistent with the data. There are conventional three methods for the job. That is the hand driven $(C D F)$, or a member of the canon of commonly used probability distributions like the exponential, normal, logistic and so on and a mixture of the conventional distributions. All these methods in one way or the other fail to satisfy all required conditions. The main objective in Powley dissertation, is to introduce Quantile-Parametrized-Distributions ( $Q P D)$. One can parameterize a QPD using over-determined systems of equations by minimizing an appropriate norm. This is done by engineering the support of a QPD using the following three methods. The first is to set up a constrained optimization problem to solve for a QPD'S coefficient. The QPD resulting from this method will pass through the extreme quantiles, but is not guaranteed to pass through the quantile-probability points. The second is to control support through truncation. When using this method, the coefficients need not be recalculated and probabilistic simulation is possible through rejection sampling. However, the resulting QPD is not guaranteed to pass through the quantile-probability points and the chopped off tails may be a poor representation of the decision maker's beliefs. The third method is to create a transformed QPD. The probability distribution resulting from this method must pass through both the extreme and intermediate quantile. In this example it is shown that the Persian-curve can be used as a replacement for all the aforementioned methods.

\section{Solution}

A set of inconsistent quantile versus probability data, selected from (Keelin and Powley, 2011) is shown in Equation (19):

$\begin{array}{lllllllll}P & 0.05 & 0.15 & 0.20 & 0.50 & 0.65 & 0.80 & 0.85 & 0.85\end{array}$

$\begin{array}{lllllllll}\lambda & 0.0 & 2.50 & 1.50 & 4.00 & 5.00 & 7.00 & 6.00 & 8.00\end{array}$
Based on the data in Equation (19), the key points and the control parameters are in Equation (20):

$O(0.0,0.0) \quad N(2.0,0.164) \quad M(4.0,0.50) \quad T(8.0,0.90)$

$a_{M}=1.25 \quad b=1$

The Persian-Fasa-curve $(P F R)$ and the PersianShiraz-curve $(P S R)$ from Equation (13), the PersianZahedan-curve $(P Z R)$ from Equation (14) and the data in Equation (19) $(Q P D)$ are shown in Fig. 6. Moreover an Upper Bound curve $(U B)$ with the key points and control parameters in Equation (21) is also shown in Fig. 6 for completeness:

$O(0.0,0.0) \quad N(2.0,0.30) \quad M(4.0,0.65) \quad T(8.0,1.0)$

$a_{M}=1.71 \quad b=0.91$

The dignity of Persian curves is highlighted in this example because the proposed functions are equivalent to the all efforts done in the cited references.

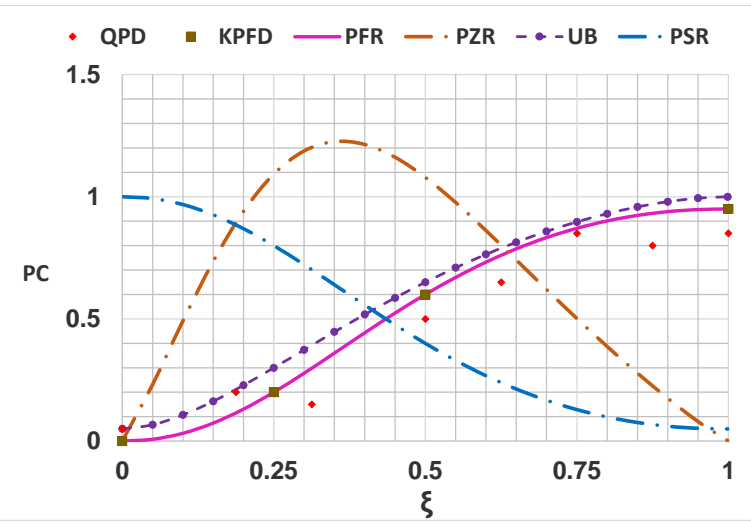

Fig. 6: Comparison of Persian curves and the keelin and powley results

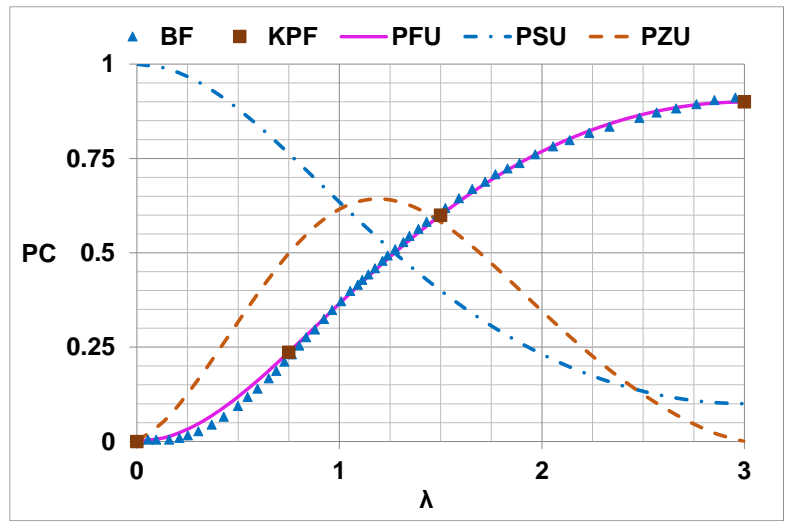

Fig. 7: Comparison of Persian curve and the baltzopoulos results 


\section{Example 2}

Compare the Persian-Fasa-curve with the lognormal probability distribution fitted on the seismic fragility data computed with the help of the SPO2FRAG software by (Baltzopoulos et al., 2017).

\section{Solution}

Baltzopoulos et al. (2017), made use of the SPO2FRAG software by (Iervolino et al., 2016), to determine the required data for construction of the fragility curve for a five story reinforced concrete building, via more than 500,000 Incremental nonlinear Dynamic Analyses (IDA). Their data are used to select key points and the control parameters in Equation (22). The Baltzopoulos curve (BF), is compared with the Persian-Fasa-curve $(P F U)$, the Persian-Shiraz-curve $(P S U)$ and the Persian-Zahedan-curve $(P Z U)$ from Equation (17) in Fig. 7. Excellent agreement of the result verified the work:

$$
\begin{aligned}
& O(0.0,0.0) \quad N(0.75,0.24) \quad M(1.5,0.60) \quad T(3.0,0.9) \\
& a_{M}=2.0 \quad b=1
\end{aligned}
$$

\section{Conclusion}

The following conclusions were obtained in the present paper.

Intensive investigation of the author's research team in the past two decades gave birth to the change of state philosophy. In this philosophy, a phenomenon is modelled as a change of state of the system between origin and destination. Origin is the threshold and the destination is the end point of the phenomenon. Via logical reasoning and mathematical logic the state of the system in each point of its lifetime is expressed in terms of two functional so called the phenomenon functions. The phenomenon functions, which are finally casted in the so called Persian curves, were then defined in terms of the state functions and two control parameters. The control parameters for a phenomenon may, simply and accurately, be obtained from the reliable or test data. The Persian curves are found to be in comply with the definition of probability functions. The proposed formulation is verified via comparison with the results of the others. The main objective of the paper is proposing a general analytical formulation for probability functions that can fit a generic dataset.

\section{Acknowledgment}

Thanks to all members of our family, specially Zahra, who provided a calm state and condition, at the time of Corona at home and encouraged writing of the paper.

\section{Author's Contributions}

Abdolrasoul Ranjbaran: Designed and contributed in writing the first draft and later corrections of the manuscript.

Mohammad Ranjbaran: Contributed in literature review, derivation of equations and engaged in the research team discussions.

Fatema Ranjbaran: Contributed in numerical analysis and engaged in the research team discussions.

\section{Ethics}

This article is original and contains unpublished material. The corresponding author confirms that all of the other authors have read and approved the manuscript and no ethical issues involved with declaration of no conflict of interest.

\section{References}

Amusan, G. E. (2010). The Beta Maxwell Distribution.

Andrews, Z. H. (2012). Continuous probability distributions in model-based specification languages (Doctoral dissertation, Newcastle University).

Adamski, K. (2014). Generalised beta type II distributions-emanating from a sequential process (Doctoral dissertation, University of Pretoria).

Adefisoye, J. O. (2015). An Assessment of the Performances of Several Univariate Tests of Normality.

Alghamdi, S. M. (2015). New and modified methods for assessing reliability equivalence (Doctoral dissertation, University of Salford).

Ali, K. A. I. (2016). A Compound Class of Lifetime Distributions (Doctoral dissertation, Cairo University).

Amirian, P., \& Ranjbaran, A. (2019). Studying the Effect of Fundamental Structural Period on the Seismic Fragility Curves of Two-Span Integral Concrete Box Girder Bridges. Iranian Journal of Science and Technology, Transactions of Civil Engineering, 1-16.

Baharvand, A., \& Ranjbaran, A. (2020a). Seismic Fragility Functions Grounded on State-Based Philosophy: Application to Low to Midrise Steel Frame Buildings. KSCE Journal of Civil Engineering, 1-12.

Baharvand, A., \& Ranjbaran, A. (2020b). A new method for developing seismic collapse fragility curves grounded on state-based philosophy. International Journal of Steel Structures, 1-17.

Baltzopoulos, G., Baraschino, R., Iervolino, I., \& Vamvatsikos, D. (2017). SPO2FRAG: Software for seismic fragility assessment based on static pushover. Bulletin of Earthquake Engineering, 15(10), 4399-4425. 
Boehm, K. (2019). Expected shortfall estimation. MSc thesis, Lund University, Sweden.

Butler, E. L. (2011). Estimating the survival distribution of aluminum processing pots.

Cui, H. (2011). Estimation of velocity distribution and suspended sediment discharge in open channels using entropy (Doctoral dissertation, Texas A \& M University).

David, F. N. (1962). Games, gods and gambling: The origins and history of probability and statistical ideas from the earliest times to the Newtonian era. Hafner Publishing Company.

Gouda, A. A. M. (2005). Probability estimation and its applications. PhD Thesis, Budapest University of Technology and Economics.

Huang, J. C. (2009). Cumulative distribution networks: Inference, estimation and applications of graphical models for cumulative distribution functions. University of Toronto.

Hao, J. (2014). Some New Probability Distributions Based on Random Extrema and Permutation Patterns.

Heinemann, C., \& Ohm, J. R. (2014). Estimation and regularization of probability density functions in image processing (No. RWTH-CONV-145199). Lehrstuhl und Institut für Nachrichtentechnik.

Hoseini, S., Ranjbaran, A., \& Roosta, S. (2018). Progressive collapse of tall steel buildings designed with outrigger braced systems. NED University Journal of Research, 15(1), 1-14.

Iervolino, I., Baltzopoulos, G., Vamvatsikos, D., \& Baraschino, R. (2016, June). SPO2FRAG v1. 0: software for PUSHOVER-BASED derivation of seismic fragility curves. In Proceedings of the VII European congress on computational methods in applied sciences and engineering, ECCOMAS, Crete Island, Greece (pp. 5-10).

Keelin, T. W., \& Powley, B. W. (2011). Quantileparameterized distributions. Decision Analysis, 8(3), 206-219.

Keelin, T. W. (2016). The metalog distributions. Decision Analysis, 13(4), 243-277.

Powley, B. W. (2013). Quantile function methods for decision analysis (Doctoral dissertation, Stanford University).

Perrone, P. (2018). Categorical Probability and Stochastic Dominance in Metric Spaces.

Ranjbaran, A. (2010). Analysis of cracked members the governing equations and exact solutions.

Ranjbaran, A., Shokrzadeh, A. R., \& Khosravi, S. (2011). A new finite element analysis of free axial vibration of cracked bars. International Journal for Numerical Methods in Biomedical Engineering, 27(10), 1611-1621.
Ranjbaran, A., \& Rousta, H. (2013). Finite element analysis of cracked beams innovative weak form equations. NED University Journal of Research, 10(1), 39-47.

Ranjbaran, A., Rousta, H., Ranjbaran, M. O., Ranjbaran, M. A., Hashemi, M., \& Moravej, M. T. (2013). A necessary modification for the finite element analysis of cracked members detection, construction and justification. Archive of Applied Mechanics, 83(7), 1087-1096.

Ranjbaran, A., \& Ranjbaran, M. (2014). New finiteelement formulation for buckling analysis of cracked structures. Journal of Engineering Mechanics, 140(5), 04014014.

Ranjbaran, A. (2014). Free-vibration analysis of stiffened frames. Journal of Engineering Mechanics, 140(9), 04014071.

Ranjbaran, A., \& Ranjbaran, M. (2016). State functions: the milestone of fracture. Archive of Applied Mechanics, 86(7), 1311-1324.

Ranjbaran, A., \& Ranjbaran, M. (2017a). State-based buckling analysis of beam-like structures. Archive of Applied Mechanics, 87(9), 1555-1565.

Ranjbaran, A., \& Ranjbaran, M. (2017b). State based damage mechanics. NED University Journal of Research, 14(1), 13-26.

Ranjbaran, A., \& Ranjbaran, M. (2018). State based method for ultimate strength analysis of stiffened panels. Ned University Journal of Research, 15(1).

Ranjbaran, A., Ranjbaran, M., \& Baharvand, A. (2020a). A step toward development of generic fragility curves. NED University Journal of Research, 17(3).

Ranjbaran, A., Ranjbaran, M., \& Ranjbaran, F. (2020b). Change of state philosophy \& Persian curves. LAP LAMBERT Academic Publishing, 225.

Satkauskas, I. V. (2017). Numerical Calculus of Probability Density Functions (Doctoral dissertation, University of Colorado at Boulder).

Weibull, W. (1951). A statistical distribution function of wide applicability. J Appl Mech, 18, 290-293.; and "discussion of the paper", Journal of Applied Mechanics, ASME. 233-234.

Williamson, R. C. (1989). Probabilistic arithmetic (Doctoral dissertation, University of Queensland).

\section{List of Symbols}

$\begin{array}{ll}a_{M}: & \text { Control parameter at } M \\ a_{N}: & \text { Control parameter at } N \\ A_{ \pm}: & \text {Sign parameter } \\ A_{ \pm}=-1: & \text { For decreasing data } \\ A_{ \pm}=+1: & \text { For increasing data } \\ b: & \text { Control parameter (power) } \\ C S P: & \text { Change of State Philosophy } \\ D: & \text { Destination function }\end{array}$




$\begin{array}{llll}E: & \text { Initial modulus } & M: & \text { Middle point } \\ F_{y}: & \text { Yield limit } & N: & \text { Next point } \\ f_{S}: & \text { System flexibility } & O: & \text { Origin (start) point } \\ f_{C}: & \text { Change flexibility } & O: & \text { Origin function } \\ F_{S}: & \text { Dimensioned flexibility } & P C: & \text { Persian curve (s) } \\ f_{S F}: & \text { Survived flexibility } & P_{O}: & \text { Origin point ordinate } \\ F_{R}: & \text { Failure function } & P_{N}: & \text { Next point ordinate } \\ \left(F_{R} \& S_{R}\right): & \text { Phenomenon functions } & P_{M}: & \text { Middle point ordinate } \\ f_{W}: & \text { Weibull probability density function } & P_{T}: & \text { End point ordinate } \\ F_{W}: & \text { Weibull cumulative distribution function } & P_{F U}: & \text { Unified Persian-Failure function } \\ \gamma: & \text { Recovery rate } & P_{S U}: & \text { Unified Persian-Survive function } \\ k_{S}: & \text { System stiffness } & P_{Z U}: & \text { Unified Persian-distribution function } \\ k_{C}: & \text { Change stiffness } & P_{Z}: & \text { Persian-Distribution function } \\ k_{S S}: & \text { Survived stiffness } & P_{F}: & \text { Persian-Failure function } \\ K P F: & \text { Key points on Failure curve } & P_{S}: & \text { Persian-Survive function } \\ K P S: & \text { Key points on Survive curve } & P_{C}=\left(P_{F} \& P_{S}\right): & \text { Persian curves } \\ K P S: & \text { Key points } & r: & \text { Effective radius of gyration } \\ L: & \text { Effective length } & R: & \text { State ratio } \\ L B: & \text { Lower bound } & S_{R}: & \text { Survive function } \\ \lambda: & \text { Lifetime parameter } & S_{F}=(D \& O): & \text { State functions } \\ \lambda=\frac{L}{r} \sqrt{\frac{F_{y}}{\pi^{2} E}}: & \text { Relative slenderness ratio } & T: & \text { Termination (end) point } \\ \lambda_{O}: & \text { Lifetime origin } & U B: & \text { Upper bound } \\ \lambda_{T}: & \text { Lifetime termination (end) } & \xi: & \text { State variable }\end{array}$

